\title{
Correction to: Use of low-molecular weight heparin, transfusion and mortality in COVID-19 patients not requiring ventilation
}

\author{
Elvira Grandone ${ }^{1,2}$ (1) $\cdot$ Giovanni Tiscia $^{1} \cdot$ Raffaele Pesavento $^{3}$ - Antonio De Laurenzo ${ }^{1} \cdot$ Davide Ceccato $^{3}$. \\ Maria Teresa Sartori ${ }^{3} \cdot$ Lucia Mirabella $^{4} \cdot$ Gilda Cinnella $^{4} \cdot$ Mario Mastroianno $^{5} \cdot$ Lidia Dalfino $^{6}$. Donatella Colaizzo ${ }^{1}$. \\ Roberto Vettor $^{3} \cdot$ Mariano Intrieri $^{7} \cdot$ Angelo Ostuni $^{8} \cdot$ Maurizio Margaglione $^{9} \cdot$ On behalf of CSS-COVID 19 Group
}

Published online: 28 April 2021

๑) Springer Science+Business Media, LLC, part of Springer Nature 2021

\section{Correction to: Journal of Thrombosis and Thrombolysis https://doi.org/10.1007/s11239-021-02429-z}

In the original version of the article, the group was mentioned incorrectly. The correct name is "CSS COVID-19 Group". Also, in the Table 4 the $\mathrm{p}$ for ICU access and CKD were 0.024 (instead of 0.24 ) and 0.006 (instead of 0.06). These have been corrected with this erratum.

Publisher's Note Springer Nature remains neutral with regard to jurisdictional claims in published maps and institutional affiliations.

The original article can be found online at https://doi.org/10.1007/ s11239-021-02429-z.

Elvira Grandone

e.grandone@operapadrepio.it

1 Thrombosis and Haemostasis Unit, Fondazione I.R.C.C.S. "Casa Sollievo della Sofferenza", Viale Cappuccini, S. Giovanni Rotondo, 71013 Foggia, Italy

2 Ob/Gyn Department of The First I.M. Sechenov Moscow State Medical University, Moscow, Russia

3 Department of Internal Medicine, University of Padua, Padua, Italy

4 Department of Anesthesia and Intensive Care, University of Foggia, Foggia, Italy
5 Scientific Direction, Fondazione I.R.C.C.S. "Casa Sollievo Della Sofferenza”, S. Giovanni Rotondo, Foggia, Italy

6 Anesthesia and Intensive Care Unit, University of Bari, Bari, Italy

7 Department of Medicine and Health Science "V. Tiberio", University of Molise, Campobasso, Italy

8 Immunohematology and Transfusion Medicine Service, Azienda Ospedaliero-Universitaria Consorziale Policlinico di Bari, University of Bari "Aldo Moro", and Struttura Regionale Coordinamento Puglia, Bari, Italy

9 Medical Genetics, University of Foggia, Foggia, Italy 